\title{
LANGUAGE MODELING FOR MULTI-DOMAIN SPEECH-DRIVEN TEXT RETRIEVAL
}

\author{
Katunobu Itou ${ }^{1}$, Atsushi Fujii ${ }^{2}$, Tetsuya Ishikawa ${ }^{2}$ \\ ${ }^{1}$ National Institute of Advanced Industrial Science and Technology \\ 1-1-1 Chuuou Daini Umezono, Tsukuba, 305-8568, Japan, E-mail: itou@ni.aist.go.jp \\ ${ }^{2}$ University of Library and Information Science \\ 1-2 Kasuga, Tsukuba, 305-8550, Japan, E-mail: \{fujii,ishikawa\}@ulis.ac.jp
}

\begin{abstract}
We report experimental results associated with speech-driven text retrieval, which facilitates retrieving information in multiple domains with spoken queries. Since users speak contents related to a target collection, we produce language models used for speech recognition based on the target collection, so as to improve both the recognition and retrieval accuracy. Experiments using existing test collections combined with dictated queries showed the effectiveness of our method.
\end{abstract}

\section{INTRODUCTION}

Automatic speech recognition, which decodes human voice to generate transcriptions, has of late become a practical technology. It is feasible that speech recognition is used in real world computerbased applications, specifically, those associated with human language. In fact, a number of speech-based methods have been explored in the information retrieval (IR) community, which can be classified into the following two fundamental categories:

- spoken document retrieval, in which written queries are used to search speech (e.g., broadcast news audio) archives for relevant speech information [i]].

- speech-driven retrieval, in which spoken queries are used to retrieve relevant textual information [2, B].

Initiated partially by the TREC- 6 spoken document retrieval (SDR) track [1], various methods have been proposed for spoken document retrieval. However, a relatively small number of methods have been explored for speech-driven text retrieval, although they are associated with numerous keyboard-less retrieval applications, such as telephone-based retrieval, car navigation systems, and user-friendly interfaces.

Barnett et al. [2] performed comparative experiments related to speech-driven retrieval, where the DRAGON speech recognition system was used as an input interface for the INQUERY text retrieval system. They used as test inputs 35 queries collected from the TREC topics and dictated by a single male speaker. Crestani [3] also used the above 35 queries and showed that conventional relevance feedback techniques marginally improved the accuracy for speech-driven text retrieval.

These above cases focused solely on improving text retrieval methods and did not address problems of improving speech recognition accuracy. In fact, an existing speech recognition system was used with no enhancement. In other words, speech recognition and text retrieval modules were fundamentally independent and were simply connected by way of an input/output protocol.

However, since most speech recognition systems are trained based on specific domains, the accuracy of speech recognition across domains is not satisfactory. Thus, as can easily be predicted, in cases of Barnett et al. [2] and Crestani [3], a speech recognition error rate was relatively high and considerably decreased the retrieval accuracy. Additionally, speech recognition with a high accuracy is crucial for interactive retrieval, such as dialog-based retrieval.

Motivated by these problems, in this paper we integrate (not simply connect) speech recognition and text retrieval to improve both recognition and retrieval accuracy in the context of speechdriven text retrieval.

Unlike general-purpose speech recognition aimed to decode any spontaneous speech, in the case of speech-driven text retrieval, users usually speak contents associated with a target collection, from which documents relevant to their information need are retrieved. In a stochastic speech recognition framework, the accuracy depends primarily on acoustic and language models $[$ ]. While acoustic models are related to phonetic properties, language models, which represent linguistic contents to be spoken, are related to target collections. Thus, it is intuitively feasible that language models have to be produced based on target collections.

To sum up, our belief is that by adapting a language model based on a target IR collection, we can improve the speech recognition and text retrieval accuracy, simultaneously.

Section 2 describes our speech-driven text retrieval system, which is currently implemented for Japanese. Section 3 elaborates on comparative experiments, in which IR test collections in different domains are used to evaluate the effectiveness of our system.

\section{SYSTEM DESCRIPTION}

\subsection{Overview}

Figure 1 depicts the overall design of our speech-driven text retrieval system, which consists of speech recognition and text retrieval modules. In the following sections, we explain two modules in Figure 1, respectively.

The first and second authors are also members of CREST, Japan Science and Technology Corporation. 


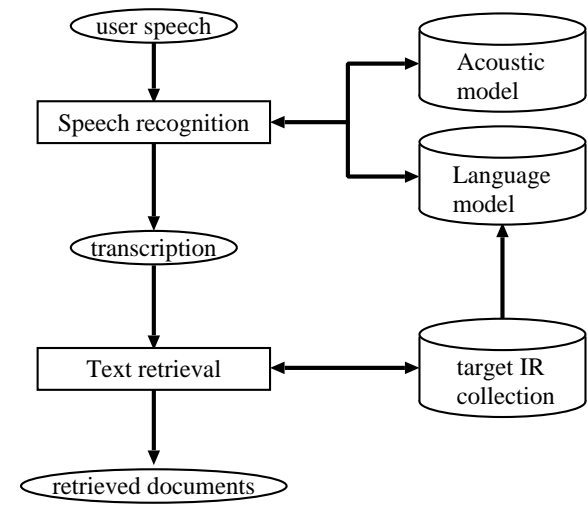

Fig. 1. The design of our speech-driven text retrieval system.

\subsection{Speech Recognition}

For the speech recognition module, we use the Japanese dictation toolkit [河], which includes the "Julius" recognition engine and acoustic/language models. Julius performs a two-pass (forwardbackward) search using word-based forward bigrams and backward trigrams on the respective passes.

The acoustic model was produced by way of the ASJ speech databases of phonetically balanced sentences (ASJ-PB) and newspaper articles texts (ASJ-JNAS) [6], which contain approximately 20,000 sentences uttered by 132 speakers including the both gender groups. We used a 16-mixture Gaussian distribution triphone Hidden Markov Model, where states were clustered into 2,000 groups by a state-tying method.

This toolkit also includes development softwares, so that acoustic and language models can be produced and replaced depending on the application. While we use the acoustic model provided in the toolkit, we use new language models produced by way of source documents (i.e., target IR collections).

\subsection{Text Retrieval}

The text retrieval module is based on the "Okapi" method [], which computes the relevance score between the transcribed query and each document in the collection, based on the distribution of index terms, and sorts retrieved documents according to the score in descending order.

We use content words extracted from documents as index terms, and perform a word-based indexing. For this purpose, we use the ChaSen morphological analyzer [8] to extract content words. We extract terms from transcribed queries using the same method.

\section{EXPERIMENTATION}

\subsection{Test Collections}

To investigate the performance of our multi-domain speech-driven retrieval system, we used two different types of Japanese IR test (benchmark) collections: the NTCIR and IREX collections. Both collections, which resemble one used in the TREC ad hoc retrieval track, include topics (information need) and relevance assessment

\footnotetext{
${ }^{1}$ http://winnie.kuis.kyoto-u.ac.jp/dictation/
}

(correct judgement) for each topic, along with target documents. However, these collections are associated with different domain, respectively.

The NTCIR collection includes 736,166 abstracts collected from technical papers published by 65 Japanese associations for various fields. On the other hand, the IREX collection 10 includes 211,853 articles collected from two years worth of "Mainichi Shimbun" newspaper articles "].

The NTCIR and IREX collections include 132 and 30 Japanese topics, respectively, for a sample of which English translations are also provided. Figures 2 and 3 show example topics in each collection, which consist of different fields (for example, descriptions and narratives) tagged in an SGML form.

Since both collections do not contain spoken queries, we asked four speakers (two males/females) to dictate topics. For this purpose, we selectively used a specific field, so as to simulate a realistic speech-driven retrieval.

In the case of the NTCIR topics, titles are not informative for the retrieval. On the other hand, narratives, which usually consist of several sentences, are too long to speak. Thus, only descriptions, which consist of a single phrase and sentence, were dictated by each speaker, so as to produce four different sets of 132 spoken queries. However, in the case of the IREX topics, since descriptions are not informative for the retrieval, only narratives were dictated by each speaker, to produce four different sets of 30 spoken queries.

\subsection{Comparative Evaluation}

We compared the performance of the following retrieval methods:

- text-to-text retrieval, which used written queries, and can be seen as the perfect speech-driven text retrieval,

- speech-driven text retrieval, in which a language model produced based on the NTCIR collection was used,

- speech-driven text retrieval, in which a language model produced based on the IREX collection was used.

In cases of speech-driven text retrieval methods, queries dictated by four speakers were used independently, and the final result was obtained by averaging results for different speakers.

Although the Julius decoder outputs more than one transcription candidates for a single speech, we used only the one with the greatest probability score. The results did not significantly change depending on whether or not we used lower-ranked transcriptions as queries.

The only difference in producing two different language models (i.e., those based on the NTCIR and IREX collections) is the source documents. In other words, both language models were of the same vocabulary size $(20,000)$, and were produced by way of the same softwares.

Table 11 shows statistics related to word tokens/types in two different collections for language modeling, where the line "Coverage" denotes the ratio of word tokens contained in the resultant language model. Most of word tokens were covered irrespective of the collection.

\footnotetext{
${ }^{2}$ http://research.nii.ac.jp/ ntcadm/index-en.html

${ }^{3} \mathrm{http}: / / \mathrm{cs}$.nyu.edu/cs/projects/proteus/irex/index-e.html

${ }^{4}$ In practice, the IREX collection provides only article IDs, which corresponds to articles in Mainichi Shimbun newspaper CD-ROM'94-'95. Participants must get a copy of the CD-ROMs themselves.
} 


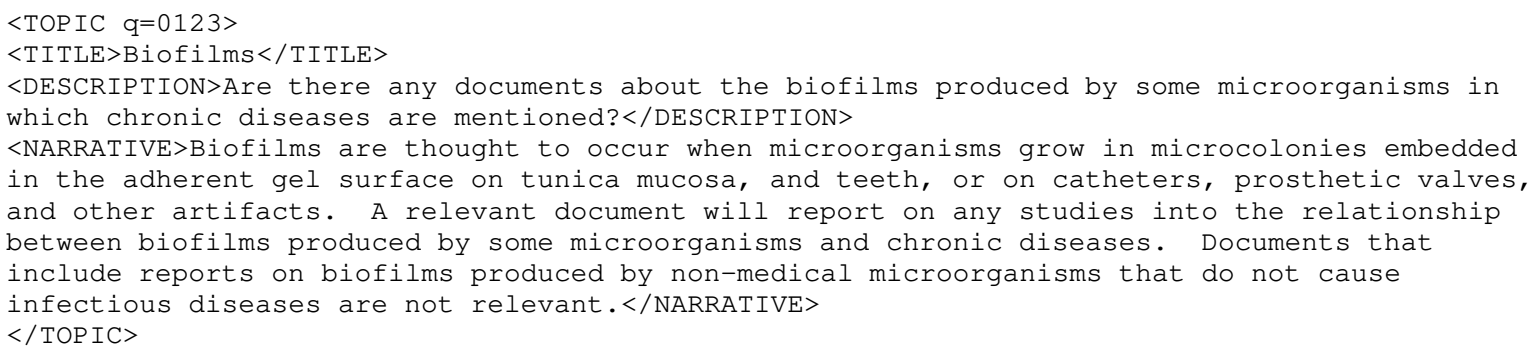

Fig. 2. An English translation for an example topic in the NTCIR collection.

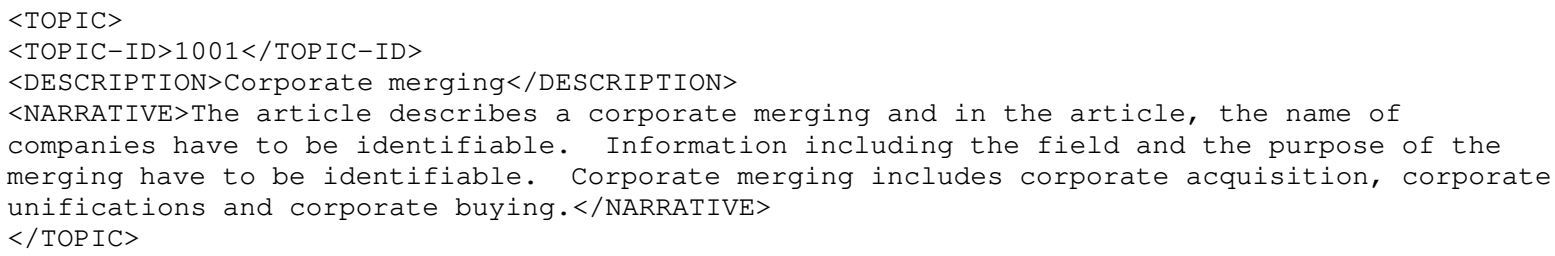

Fig. 3. An English translation for an example topic in the IREX collection.

Table 1. Statistics related to source words for language modeling.

\begin{tabular}{lcc}
\hline & NTCIR & IREX \\
\hline \# of Types & $454 \mathrm{~K}$ & $179 \mathrm{~K}$ \\
\# of Tokens & $175 \mathrm{M}$ & $53 \mathrm{M}$ \\
Coverage & $97.9 \%$ & $96.5 \%$ \\
\hline
\end{tabular}

Each method retrieved 1,000 top documents, and the TREC evaluation software was used to calculate non-interpolated average precision values and plot recall-precision curves.

Table 2 shows the non-interpolated average precision values (AP) and word error rate in speech recognition, for different retrieval methods. As with existing experiments for speech recognition, word error rate (WER) is the ratio between the number of word errors (i.e., deletion, insertion, and substitution) and the total number of words. In addition, we investigated error rate with respect to query terms (i.e., keywords used for retrieval), which we shall call "term error rate (TER)". Table 2 also shows trigram test-set perplexity (PP) and test-set out-of-vocabulary rate (OOV).

It should noted that for all the evaluation measures in Table 2 excepting average precision, smaller values are generally obtained with better methods. Suggestions which can be derived from these results are as follows.

First, by comparing results of different language models, one can see that the performance was significantly improved with a language model produced from the target collection, which was observable irrespective of the domain. Thus, producing language models based on target collections was quite effective for speechdriven text retrieval.

Second, while in the case of the NTCIR collection, the average precision for speech-driven retrieval was approximately $77 \%$ of that obtained with text-to-text retrieval, in the case of the IREX collection, the average precision for speech-driven retrieval was quite comparable that obtained with text-to-text retrieval.

Third, TER was generally higher than WER irrespective of the speaker. In other words, speech recognition for content words was more difficult than functional words, which were not contained in query terms.

Finally, we investigated the trade-off between recall and precision. Figures 4 and 5 show recall-precision curves of different retrieval methods, for the NTCIR and IREX collections, respectively. In these figures, the relative superiority for precision values due to different language models in Table 6 was also observable, regardless of the recall.

\section{CONCLUSION}

Aiming at speech-driven text retrieval with a high accuracy, we proposed a method to integrate speech recognition and text retrieval methods, in which target text collections are used to produce statistical language models for speech recognition. We also showed the effectiveness of our method by way of experiments, where dictated information needs in the NTCIR/IREX collections were used as queries to retrieve documents in different domains.

\section{Acknowledgments}

The authors would like to thank the National Institute of Informatics for their support with the NTCIR collection and the IREX committee for their support with the IREX collection.

\section{REFERENCES}


Table 2. Results for different retrieval methods targeting the NTCIR/IREX collections (AP: average precision, WER: word error rate, TER: term error rate, PP: trigram test-set perplexity, OOV: test-set Out-of-Vocabulary rate).

\begin{tabular}{|c|c|c|c|c|c|c|c|c|c|c|}
\hline \multirow[b]{2}{*}{ Language Mode } & \multicolumn{5}{|c|}{ NTCIR } & \multicolumn{5}{|c|}{ IREX } \\
\hline & $\mathrm{AP}$ & WER & TER & $\mathrm{PP}$ & $\mathrm{OOV}$ & $\mathrm{AP}$ & WER & TER & $\mathrm{PP}$ & OOV \\
\hline Text & 0.337 & - & - & - & - & 0.367 & - & - & - & - \\
\hline NTCIR & 0.261 & $18.6 \%$ & $23.6 \%$ & 60 & $4.2 \%$ & 0.166 & $31.1 \%$ & $41.0 \%$ & 138 & $6.1 \%$ \\
\hline IREX & 0.111 & $41.4 \%$ & $54.6 \%$ & 195 & $9.4 \%$ & 0.334 & $19.5 \%$ & $22.9 \%$ & 108 & $1.4 \%$ \\
\hline
\end{tabular}

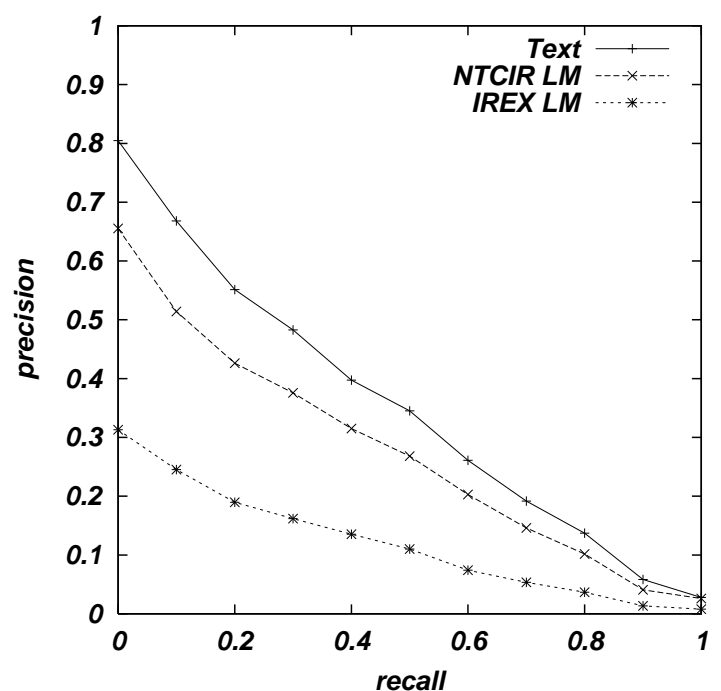

Fig. 4. Recall-precision curves for different methods targeting the NTCIR collection.

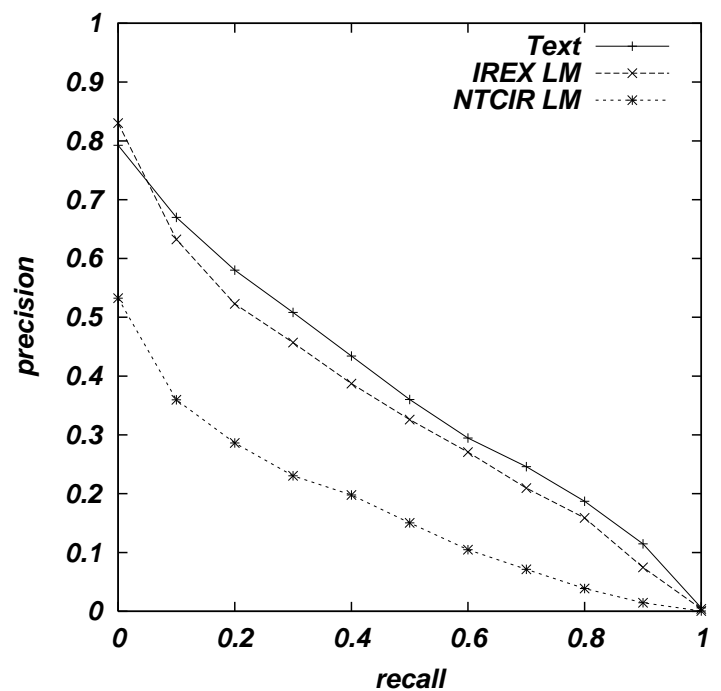

Fig. 5. Recall-precision curves for different methods targeting the IREX collection.
[1] John S. Garofolo, Ellen M. Voorhees, Vincent M. Stanford, and Karen Sparck Jones, "TREC-6 1997 spoken document retrieval track overview and results," in Proceedings of the 6th Text REtrieval Conference, 1997, pp. 83-91.

[2] J. Barnett, S. Anderson, J. Broglio, M. Singh, R. Hudson, and S. W. Kuo, "Experiments in spoken queries for document retrieval," in Proceedings of Eurospeech97, 1997, pp. 13231326.

[3] Fabio Crestani, "Word recognition errors and relevance feedback in spoken query processing," in Proceedings of the Fourth International Conference on Flexible Query Answering Systems, 2000, pp. 267-281.

[4] Lalit. R. Bahl, Frederick Jelinek, and Robert L. Mercer, "A maximum linklihood approach to continuous speech recognition," IEEE Transactions on Pattern Analysis and Machine Intelligence, vol. 5, no. 2, pp. 179-190, 1983.

[5] T. Kawahara, A. Lee, T. Kobayashi, K. Takeda, N. Minematsu, S. Sagayama, K. Itou, A. Ito, M. Yamamoto, A. Yamada, T. Utsuro, and K. Shikano, "Free software toolkit for Japanese large vocabulary continuous speech recognition," in Proceedings of the 6th International Conference on Spoken Language Processing, 2000, pp. 476-479.

[6] K. Itou, M. Yamamoto, K. Takeda, T. Takezawa, T. Matsuoka, T. Kobayashi, K. Shikano, and S. Itahashi, "The design of the newspaper-based Japanese large vocabulary continuous speech recognition corpus," in ICSLP-98, 1998, pp. 3261-3264.

[7] S. E. Robertson and S. Walker, "Some simple effective approximations to the 2-poisson model for probabilistic weighted retrieval," in Proceedings of the 17th Annual International ACM SIGIR Conference on Research and Development in Information Retrieval, 1994, pp. 232-241.

[8] Yuji Matsumoto, Akira Kitauchi, Tatsuo Yamashita, Yoshitaka Hirano, Hiroshi Matsuda, and Masayuki Asahara, "Japanese morphological analysis system ChaSen version 2.0 manual 2nd edition," Tech. Rep. NAIST-IS-TR99009, NAIST, 1999.

[9] National Institute of Informatics, Proceedings of the 2nd NTCIR Workshop Meeting on Evaluation of Chinese \& Japanese Text Retrieval and Text Summarization, 2001.

[10] Satoshi Sekine and Hitoshi Isahara, "IREX: IR and IE evaluation project in Japanese," in Proceedings of the 2nd International Conference on Language Resources and Evaluation, 2000, pp. 1475-1480. 